\title{
Brolucizumab-key learnings from HAWK and HARRIER
}

\author{
Ashish Sharma ${ }^{1} \cdot$ Nikulaa Parachuri $^{1} \cdot$ Nilesh Kumar $^{1}{ }^{1} \cdot$ Rohini Sharma $^{2} \cdot$ Francesco Bandello $^{3}$. \\ Baruch D. Kuppermann ${ }^{4}$ - Anat Loewenstein ${ }^{5}$
}

Received: 21 February 2020 / Accepted: 2 March 2020 / Published online: 13 March 2020

(c) The Royal College of Ophthalmologists 2020

Anti-vascular endothelial growth factor (anti-VEGF) therapy has revolutionized the management of neovascular agerelated macular degeneration (nAMD) in the last few years $[1,2]$. Though there are various anti-VEGF molecules already in use for the management of nAMD (Bevacizumab (Avastin, Genentech Inc, USA), Ranibizumab (Lucentis, Genentech Inc, USA), Aflibercept (Eylea, Regeneron Pharmaceuticals Inc, USA), and the biosimilar Razumab (Intas Pharmaceuticals Ltd, Ahmedabad, GJ, India)), Recently U.S. Food and Drug Administration has granted approval for another anti-VEGF, brolucizumab (Beovu, Novartis) [3, 4]. At present, the molecule is approved for clinical use in the USA for the management of nAMD. Brolucizumab has received final approval on the basis of outcomes of the two major phase 3 clinical trials-HAWK and HARRIER [5]. The major differences between the two trials were the population inclusion of the USA in HAWK trial and exclusion in HARRIER, and HAWK trial included two doses of brolucizumab 3 and $6 \mathrm{mg}$ while HARRIER compared only $6 \mathrm{mg}$. Based on the results of HAWK and HARRIER trials, the committee for medicinal products for human use (CHMP) has adopted a positive opinion for $6 \mathrm{mg}$ brolucizumab, which will form the basis for European

$\triangle$ Ashish Sharma

drashish79@hotmail.com

1 Lotus Eye Hospital and Institute, Avinashi Road, Coimbatore, TN, India

2 Department of Healthcare, The TIPS Global Institute, Coimbatore, Tamil Nadu, India

3 University Vita-Salute, Scientific Institute San Raffaele, Milano, Italy

4 Gavin Herbert Eye Institute, University of California, Irvine, CA, USA

5 Division of Ophthalmology, Tel Aviv Sourasky Medical Center and Sackler Faculty of Medicine, Tel Aviv University, Tel Aviv, Israel medicines agency's (EMA's) decision on marketing approval. The EMA's decision is expected to be made in the first half of 2020 [6]. Furthermore, brolucizumab is expected to be approved for clinical use in other countries by the year 2020. It is important for clinicians to understand the key outcomes of HAWK and HARRIER before they start using it in clinical practice.

The study was a 96-week prospective, randomized, double-masked, multi-centre trial with 1800 patients enrolled from 400 centres around the world. They were designed to compare the efficacy and safety of intravitreal injections of brolucizumab vs $2 \mathrm{mg}$ aflibercept. The design of these studies was formulated after analyses from multiple previous studies such as CATT, OSPREY, PIER, and EXCITE [7-10].

Patients $\geq 50$ years who had untreated, active choroidal neovascularization lesions secondary to AMD affecting the central subfield having best-corrected visual acuity (BCVA) between Snellen equivalents $\approx 20 / 32$ to $20 / 400$ with no fibrosis or geographic atrophy affecting the central subfield were included in the study. Eyes were randomized 1:1:1 to brolucizumab $3 \mathrm{mg}$, brolucizumab $6 \mathrm{mg}$, or aflibercept $2 \mathrm{mg}$ (HAWK) or $1: 1$ to brolucizumab $6 \mathrm{mg}$ or aflibercept $2 \mathrm{mg}$ (HARRIER). Following the 3-month loading phase (weeks 0,4 , and 8), patients in the brolucizumab arms received a $\mathrm{q} 12 \mathrm{w}$ dosing interval. They were shifted to a $\mathrm{q} 8 \mathrm{w}$ dosing interval if disease activity was noted. Aflibercept was dosed bi-monthly at the time of study initiation. Disease activity assessments were done at weeks 20,32, and 44. Additional assessments were performed 8 weeks after every scheduled q12w brolucizumab injection in the HARRIER trial.

A protocol review (BCVA decline) was done at week 16 to identify patients who had a higher anti-VEGF treatment need. Patients underwent a complete ophthalmic exam which included BCVA, SD-OCT (IRF (intraretinal fluid)/ SRF (subretinal fluid)/sub-RPE (retinal pigment epithelium) fluid and central subfield thickness (CST)). Patients were evaluated for adverse events every 4 weeks in both the studies. 


\section{Key outcomes}

\section{BCVA}

The primary outcome was mean BCVA change from baseline to week 48. Secondary endpoints were BCVA change from baseline averaged over the period of week 36 through week 48 , q12w treatment status at week 48 (brolucizumab only), and $\mathrm{q} 12 \mathrm{w}$ treatment status at week 48 among eyes with no q8w need during the first $\mathrm{q} 12 \mathrm{w}$ cycle, change in BCVA, CST, SRF, sub-RPE fluid, and IRF from the baseline and the presence of disease activity at week 16 .

In HAWK, $91.4 \%, 89.8 \%$, and $87.3 \%$ of patients treated with brolucizumab 3, 6, and aflibercept, respectively, completed the study treatment up to week 48. In HARRIER, 93.3 and $93.5 \%$ of brolucizumab $6 \mathrm{mg}$ and aflibercept-treated patients, respectively, completed study treatment up to week 48. Mean baseline BCVA was 60.6 letters in HAWK and 61.2 in HARRIER and $\approx 25 \%$ of study eyes had BCVA $\geq 71$ letters at baseline.

In both trials, with respect to BCVA, each brolucizumab arm demonstrated noninferiority to aflibercept. In HAWK, brolucizumab $3 \mathrm{mg}$ and brolucizumab $6 \mathrm{mg}$ treated eyes gained +6.1 and +6.6 letters, respectively, vs +6.8 letters of aflibercept treated eyes. In HARRIER, brolucizumab 6 mg treated eyes gained +6.9 letters vs +7.6 letters of aflibercept treated eyes. $25.2 \%$ of the study eyes in the brolucizumab $3 \mathrm{mg}$ arm, 33.6\% in the brolucizumab $6 \mathrm{mg}$ arm, and $25.4 \%$ in the aflibercept $2 \mathrm{mg}$ gained $\geq 15$ letters of vision from baseline to week 48 in HAWK. 29.3 and 29.9\% of the brolucizumab $6 \mathrm{mg}$ and aflibercept $2 \mathrm{mg}$ gained $\geq 15$ letters of vision from baseline to week 48 , respectively, in HARRIER.

\section{Maintenance of $q 12$ dosing}

In the HAWK trial, $49 \%$ and $56 \%$ of patients receiving 3 and $6 \mathrm{mg}$ brolucizumab, respectively, maintained their q12 week dosing interval through Week 48. In the HARRIER trial, $51 \%$ of eyes receiving $6 \mathrm{mg}$ brolucizumab maintained a q12 week dosing interval. In both trials, about $50 \%$ of the patients were able to maintain their q12 week dosing schedule.

\section{Disease activity}

Anatomical outcomes were measured to assess the disease activity which was done at week 16 . Both studies showed that at week 16, brolucizumab demonstrated much better anatomical outcomes than aflibercept. In the HAWK trial, the CST reduction was greater among eyes treated with brolucizumab $6 \mathrm{mg}$ vs aflibercept (161.4 vs $-133.6 \mu \mathrm{m}$.) and HARRIER (174.4 vs $-134.2 \mu \mathrm{m})$. IRF/SRF was present in fewer brolucizumab-treated eyes vs aflibercepttreated eyes at week 16 in HAWK, 3 mg (41.8 vs 52.0\%) and $6 \mathrm{mg}$ (33.9 vs 52.2\%) and in HARRIER (29.4 vs $45.1 \%$ ). Sub-RPE fluid was present in fewer brolucizumab $6 \mathrm{mg}$ treated eyes than aflibercept treated eyes at week 48 in HAWK (13.5\% vs $21.6 \%)$ and HARRIER (12.9 vs $22.0 \%)$

\section{Safety}

The safety and side effect profile of brolucizumab was similar to aflibercept. Ocular adverse events in $<5 \%$ of the patients included decreased visual acuity, conjunctival and retinal haemorrhage, vitreous floaters, eye pain, dry eye, cataract, and vitreous detachment.

Brolucizumab met the primary and secondary endpoints at weeks 48 and 96 and proved non-inferiority to aflibercept. To summarize, brolucizumab seems to be a promising novel anti-VEGF molecule as per the key outcomes of the HAWK and HARRIER trials, such as maintenance of BCVA on $\mathrm{q} 12 \mathrm{w}$ regimen and better control of disease activity. Real-world outcomes are yet to be seen.

\section{Compliance with ethical standards}

Conflict of interest AS: Consultant: for Novartis, Allergan, Bayer and Intas. FB: Consultant: Allergan, Bayer, Boehringer-Ingelheim, Fidia Sooft, Hofmann La Roche, Novartis, NTC Pharma, Sifi, Thrombogenics, Zeiss. BDK: Clinical research: Alcon, Alimera, Allegro, Allergan, Apellis, Clearside, Genentech, GSK, Ionis, jCyte, Novartis, Regeneron, ThromboGenics; CONSULTANT: Alimera, Allegro, Allergan, Cell Care, Dose, Eyedaptic, Galimedix, Genentech, Glaukos, Interface Biologics, jCyte, Novartis, Ophthotech, Regeneron, Revana, Theravance Biopharma. AL reports other from Allergan, other from Novartis, other from Roche, other from Notal Vision, other from Forsightslabs, other from Beyeonics, other from Bayer Health Care. NK: None. NP: None. RS: None.

Publisher's note Springer Nature remains neutral with regard to jurisdictional claims in published maps and institutional affiliations.

\section{References}

1. Brown DM, Michels M, Kaiser PK, Heier JS, Sy JP, Ianchulev T. Ranibizumab versus verteporfin photodynamic therapy for neovascular age-related macular degeneration: two-year results of the ANCHOR study. Ophthalmology 2009;116:57-65.e5.

2. Mitchell P, Bressler N, Doan QV, Dolan C, Ferreira A, Osborne A, et al. Estimated cases of blindness and visual impairment from neovascular age-related macular degeneration avoided in australia by ranibizumab treatment. PLoS ONE 2014;9:e101072.

3. Sharma A, Reddy P, Kuppermann BD, Bandello F, Lowenstein A. Biosimilars in ophthalmology: "Is there a big change on the horizon?". Clin Ophthalmol. 2018;12:2137-43.

4. Jerry H. Brolucizumab gets FDA approval for wet AMD. https://www. retinalphysician.com/issues/2019/october-2019/brolucizumab-getsfda-approval-for-wet-amd. Accessed 7 Feb 2020.

5. Dugel PU, Koh A, Ogura Y, Jaffe GJ, Schmidt-Erfurth U, Brown DM, et al. HAWK and HARRIER: phase 3, multicenter, 
randomized, double-masked trials of brolucizumab for neovascular age-related macular degeneration. Ophthalmology 2020;127: 72-84.

6. Beovu (brolucizumab). https://www.ema.europa.eu/en/medicines/ human/summaries-opinion/beovu. Accessed 7 Feb 2020.

7. CATT Research Group, Martin DF, Maguire MG, Ying GS, Grunwald JE, Fine SL, Jaffe GJ. Ranibizumab and bevacizumab for neovascular age-related macular degeneration. N. Engl J Med. 2011;364:1897-908.

8. Regillo CD, Brown DM, Abraham P, Yue H, Ianchulev T, Schneider $\mathrm{S}$, et al. Randomized, double-masked, sham-controlled trial of ranibizumab for neovascular age-related macular degeneration: PIER Study year 1. Am J Ophthalmol. 2008;145: 239-48.

9. Schmidt-Erfurth U, Eldem B, Guymer R, Korobelnik JF, Schlingemann RO, Axer-Siegel R, et al. Efficacy and safety of monthly versus quarterly ranibizumab treatment in neovascular age-related macular degeneration: the EXCITE study. Ophthalmology 2011;118:831-9.

10. Yannuzzi NA, Freund KB. Brolucizumab: evidence to date in the treatment of neovascular age-related macular degeneration. Clin Ophthalmol. 2019;13:1323-1329. 